\title{
3D Nanoprinting via Focused Electron Beams
}

Harald Plank ${ }^{1,2}$, Robert Winkler ${ }^{1,2}$, Jürgen Sattelkow ${ }^{1}$, Jason D. Fowlkes ${ }^{3,4}$, Philip D. Rack ${ }^{3,4}$

${ }^{1 .}$ Institute of Electron Microscopy and Nanoanalysis, Graz University of Technology, Graz, Austria

2. Graz Centre for Electron Microscopy, Graz, Austria

3. Centre for Nanophase Materials Sciences, Oak Ridge National Laboratories, Oak Ridge, TN, United States

4. Department for Material Sciences, University of Tennessee, Knoxville, TN, United States

3D-printing of functional structures has emerged as an important technology in research and development. While being reliable on the micro and sub-micron scale, the extension to the nanoscale is still a challenging task. Among the very few direct-write techniques on that scale, focused electron beam induced deposition (FEBID) is one of the promising candidates as this technology allows fabrication of functional nanostructures on almost any material and substrate morphology in a single-step process. Based on strong fundamental progress in recent years, FEBID was demonstrated to be capable of fabricating complex, freestanding 3D nano-architectures with individual branch diameters down to $20 \mathrm{~nm}[1,2]$. Together with the increasing availability of precursors with different functionalities, FEBID is advancing from a versatile research tool into a predictable and reliable 3D nano-printer, which opens up new opportunities for advanced applications.

In this contribution, we start with the basic principles of 3-dimensional printing via FEBID, complemented by simulations for deeper insight into the fundamental processes that are operative [3]. Next, we briefly introduce a recently released computer aided design software (3BID) [4], which allows the reliable and easy design of complex 3D objects (see Figure 1). In the following, we present a variety of 3BID based proof-of-principle studies to demonstrate the capabilities of this direct-write technology. This ranges from scientifically oriented applications, such as plasmonics [1], magnetics [5] and nano-mechanics [6] toward industrially relevant concepts for scanning probe microscopy related tip fabrication, such as electrical, thermal and optical 3D nano-probes (see Figure 2). Finally, we overview some of the remaining challenges and provide an outlook on future activities.

\section{References:}

[1] R Winkler et al, ACS Appl. Mater. Interfaces 9 (2017), p. 8233.

[2] JD Fowlkes et al, ACS Nano 10 (2016), p. 6163.

[3] R Winkler et al, ACS Appl. Nano Mater. (2018), in press.

[4] JD Fowlkes et al, ACS Appl. Nano Mater. (2018), in press.

[5] M Al Mamoori et al, Materials (Basel) 11 (2018), p. 289.

[6] G Arnold et al, Adv. Funct. Mater. (2018), in press. 


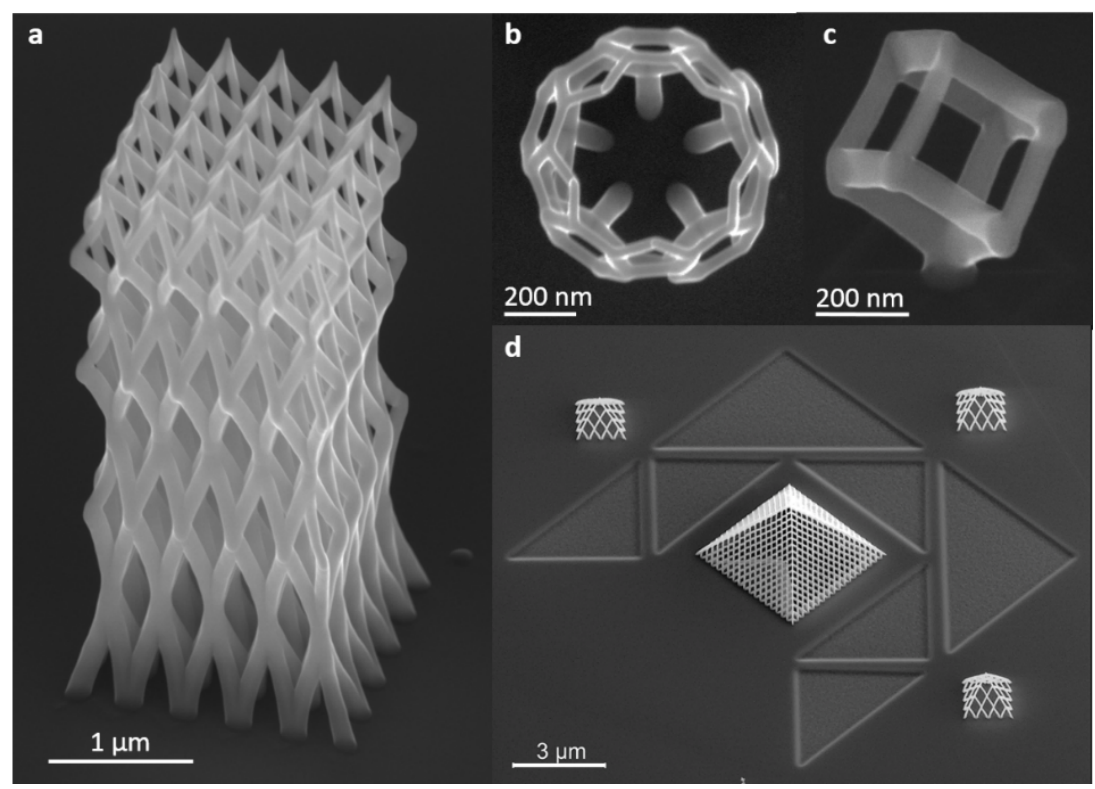

Figure 1. Direct-write 3D nano-printing of meshed-like platinum-carbon 3D nano-architectures: (a) sponge tower, (b) open Buckyball, (c) i-cube, (d) 3BID-model of the glass pyramid of the Louvre in Paris on a FIB-pre-structured silicon substrate. All images are SEM side views except (b), which is imaged from top.
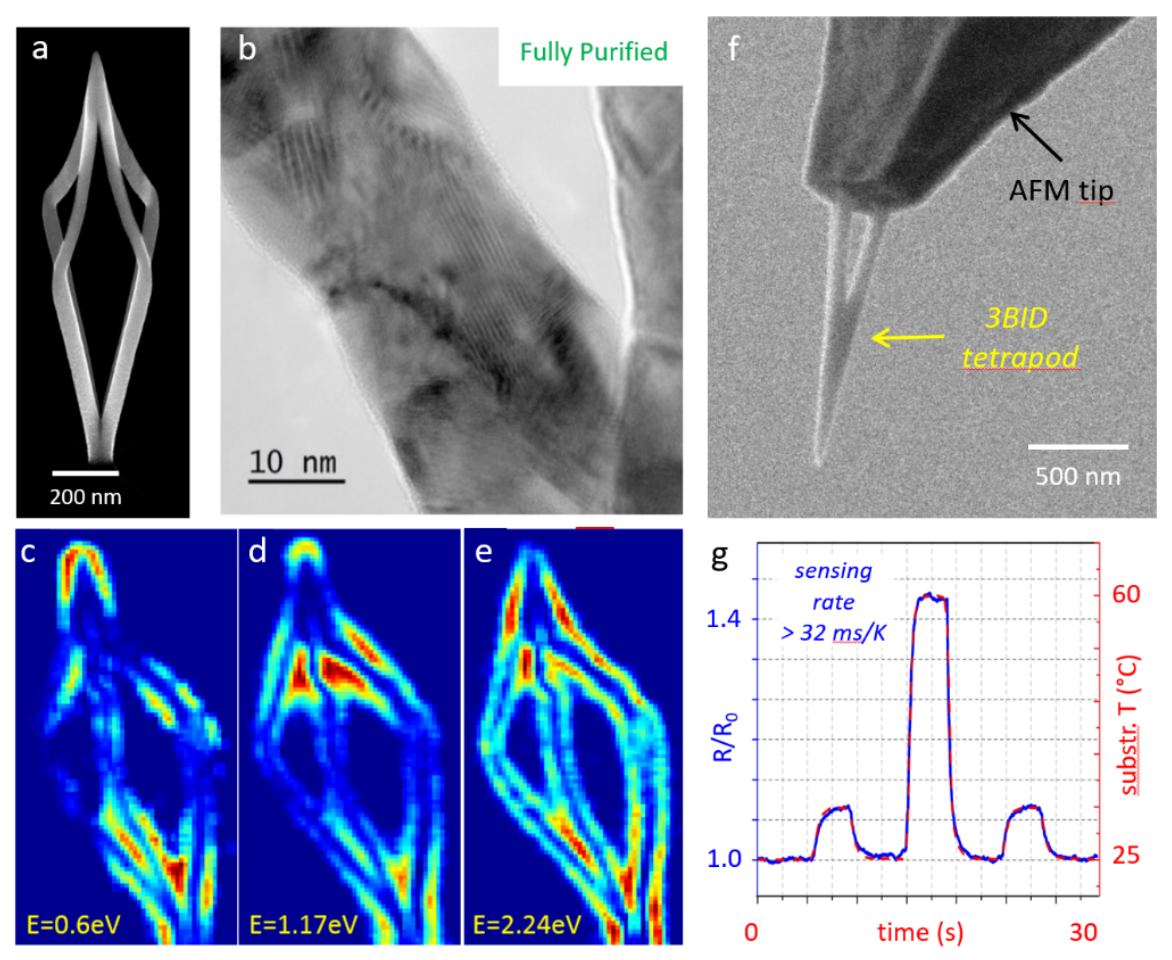

Figure 2. 3D structures for plasmonic (a-e) and thermal nano-probing (f-g). (a) shows a freestanding AuC 3D structure which is transformed into pure Au via purification (b). (c-e) STEM-EELS characterization at different energies, which confirm plasmonic activities. (f) 3BID tetrapod on top of a FIB pre-processed self-sensing cantilever for further application as thermal nano-probe. ( $g$ ) shows the timely response by electric current readout through the $3 \mathrm{D}$ tetrapod revealing sensing rates of $32 \mathrm{~ms} / \mathrm{K}$. 